\title{
De « classe ouvrière » à " classes moyennes ", une réfection terminologique du champ social en
}

\section{France}

From "Working Class" to "Middle Classes", a Lexical Turn in the Social Field in France

\section{Marc Arabyan}

\section{OpenEdition \\ Journals}

Édition électronique

URL : http://journals.openedition.org/pratiques/2327

DOI : $10.4000 /$ pratiques.2327

ISSN : 2425-2042

Éditeur

Centre de recherche sur les médiations (CREM)

Édition imprimée

Date de publication : 31 décembre 2014

Référence électronique

Marc Arabyan, "De "classe ouvrière » à "classes moyennes », une réfection terminologique du champ social en France », Pratiques [En ligne], 163-164 | 2014, mis en ligne le 31 décembre 2014, consulté le 14 novembre 2019. URL : http://journals.openedition.org/pratiques/2327 ; DOI : 10.4000/ pratiques. 2327

Ce document a été généré automatiquement le 14 novembre 2019.

(c) Tous droits réservés 


\title{
De « classe ouvrière » à « classes moyennes ", une réfection terminologique du champ social en France
}

\author{
From "Working Class" to "Middle Classes", a Lexical Turn in the Social Field in
}

France

Marc Arabyan

There's class warfare, all right, Mr. Buffett said, but it's my class, the rich class, that's making war, and we're winning.

Ben Stein, "In class warfare, guess which class is winning," New York Times, 26.11.06.

\section{Introduction}

1 Le rapport des médias à la vérité est une des formes du rapport du langage à la morale (Paveau, 2013), et c'est dans ce champ de connaissance qu'à la demande de Marie-Anne Paveau, qui m'a entendu m'interroger à ce sujet, je voudrais reprendre la problématique en partant de publications récentes.

2 Le «traitement médiatique» de l'actualité est une source ininterrompue d'informations sur le fonctionnement du langage, et pour ce qui concerne les médias français, voire francophones, sur le fonctionnement du français et la circulation des discours dans les pays où l'on parle cette langue. Il se prête donc particulièrement à l'analyse des discours circulants dans le corps social et dans cette partie du flux langagier qu'on appelle l'« opinion publique ». Cette opinion publique elle-même se confond peu ou prou avec le contenu des «journaux » de la presse écrite, de la radio et 
de la télévision (les fameux «JT» ou « 20 heures») qui reprennent massivement le même programme d'actualités fourni par les agences de presse.

Suivant avec intérêt depuis près de 25 ans les travaux de D. Da Cunha, dont la thèse (1992) sur la circulation de la parole dans le champ social à partir des médias a marqué la recherche sur le sujet, j'ai longtemps réfléchi sans rien écrire sur ce que pourrait être l'approche du discours des médias comme "discours massivement rapporté » d'« éléments de langage " élaborés par un petit nombre d'autorités (au sens d' auctoritates, "les auteurs») placées en amont des agences de presse, situation qui leur assure le contrôle de l'information.

L'expression "journalisme d'investigation ", apparue après le scandale du Watergate (1972-1974) qui couta son siège au président Nixon, dessine en creux l'existence d'un «journalisme de transmission » qui n'élabore pas lui-même ce qu'il rapporte.

Le fait que l'information soit médiatisée - c'est-à-dire rapportée par les uns (les journalistes) et contrôlée par les autres (les autorités) - pose deux questions: 1) L'information est-elle véridique, vérifiée (avérée au sens premier du mot) et, dans le cas contraire, peut-elle être délibérément trompeuse, c'est-à-dire mensongère ?2) dans quelle mesure les médias sont-ils corrompus?

On connait à ce sujet la célèbre déclaration du PDG de TF1, Patrick Le Lay, selon qui,

«il y a beaucoup de façons de parler de la télévision. Mais dans une perspective “business", soyons réaliste : à la base, le métier de TF1, c'est d'aider Coca-Cola, par exemple, à vendre son produit [...]. Pour qu'un message publicitaire soit perçu, il faut que le cerveau du téléspectateur soit disponible. Nos émissions ont pour vocation de le rendre disponible : c'est-à-dire de le divertir, de le détendre pour le préparer entre deux messages. Ce que nous vendons à Coca-Cola, c'est du temps de cerveau humain disponible [...]. Rien n'est plus difficile que d'obtenir cette disponibilité. C'est là que se trouve le changement permanent. Il faut chercher en permanence les programmes qui marchent, suivre les modes, surfer sur les tendances, dans un contexte où l'information s'accélère, se multiplie et se banalise » ("Le Lay (TF1) vend "du temps de cerveau humain disponible" ", in: Les Dirigeants face au changement, Paris, Éd. du Huitième Jour, repris par une dépêche AFP du 09/07/04, reprise par Libération, 10-11/07/04): "Patrick Le Lay, décerveleur " puis par Acrimed, 11/07/0411).

7 Ce type de déclaration a un effet dévastateur sur le public, mais pas suffisamment pour remettre le système médiatique en cause. Tout se passe comme si ce même public oubliait très vite les déchirures du voile qui couvre habituellement les turpitudes des médias, se contentant de ne pas les croire davantage que les hommes (et femmes) politiques :

« $72 \%$ des Français n'ont pas confiance dans l'Assemblée nationale, $73 \%$ dans le Sénat. Pour $88 \%$ des personnes interrogées, les hommes et les femmes politiques ne s'occupent pas de ce que pensent les gens. Les médias sont très vivement critiqués : $77 \%$ des personnes interrogées ne leur font pas confiance. Pour $74 \%$ des Français, les journalistes ne parlent pas des vrais problèmes des Français. [...] Pour $65 \%$ des Français, la plupart des hommes et des femmes politiques sont corrompus. $84 \%$ pensent que les hommes politiques agissent principalement pour leurs intérêts personnels. La progression [par rapport à janvier 2013] la plus spectaculaire concerne l'idée selon laquelle "le système démocratique fonctionne mal, mes idées ne sont pas bien représentées" (+ 6 points à $78 \%$ ) " (Rapport Ipsos/Steria pour Le Monde/France Inter/Cevipof/Fondation Jean-Jaurès, 21/01/142).

8 Rapportés aux tranches de revenus de la population française (voir infra, section 1), ces pourcentages suggèrent que plus on descend dans l'échelle sociale, plus le rejet culturel 
des classes dirigeantes par l'électorat est massif, mais sans se traduire autrement qu'en abstention et en vote pour le Front national (FN). C'est du moins ce qu'on peut déduire du score des élections européennes de 2014 rapporté à l'ensemble du corps électoral : 46544712 électeurs inscrits; 26796819 absentions (soit 57,57\%)； 4712461 votes FN (soit $10 \%$ ) ; total abstentions + FN : 67,57 \% (source : ministère de l'Intérieur).

Reformulée par Pierre Bourdieu entre sociologie et science politique, la situation se résume ainsi : « Les dominés n'ont aucune prise sur les médias. Il n'y a [...] de politique qu'au sein du champ du pouvoir, réservé aux classes dominantes, tandis que la majorité macère à moitié hébétée dans les marécages de la soumission, manipulée par ses porteparole » (Burawoy, 2014).

Il y a en l'occurrence une contradiction flagrante entre une analyse sociologique savante, qui voit dans l'opinion publique une masse de « dominés » trompés avec succès par les dominants, et plus des deux tiers de l'électorat qui soit s'abstiennent, soit se tournent vers un parti populiste qui passe son temps à dénoncer les élites et les règles en cours du jeu politique.

11 La critique de l'idéologie comme fausse conscience, construction illusoire de la réalité inspirée par une situation sociale, est trop complexe pour être abordée dans une discussion sur le rapport des médias à la vérité. Il semble cependant possible de partir de l'idée que l'articulation entre langage et morale se situe dans le fait de mentir ou non à celui, celle ou ceux à qui l'on s'adresse - j'entends mentir en connaissance de cause, dans l'intention de tromper (la voluntas fallendi d'A. d'Hippone dans Du mensonge, voir Weinrich, 2014: 32), comme on peut supposer que fait le journalisme de transmission.

\section{De la « sociologie de Chicago " à la « sociologie de presse »}

C'est dans cet esprit que je propose d'observer l'absorption, dans la grande presse française, du syntagme classe ouvrière par l'expression classes moyennes. Le phénomène date d'une quarantaine d'années. Il est contemporain du renoncement à la lutte des classes par le XXIIIe congrès du Parti communiste français (09-13 mai 1979), de l'arrivée au pouvoir de Margaret Thatcher au Royaume-Uni (04 mai 1979), suivie de celles de Ronald Reagan aux États-Unis (20 janvier 1981) et de François Mitterrand en France (21 mai 1981). Ces évènements marquent le triomphe de l'École de Chicago ${ }^{3}$ et le retour du libéralisme comme principe politique, accompagné d'une sociologie désormais dominante qui analyse les classes en termes de revenus et non plus de positions sociales conflictuelles (Jacoby, 2014). Par commodité, faute de mieux, j'appellerai cette sociologie la « sociologie de Chicago ».

Mon hypothèse est qu'il ne s'agit pas d'une coïncidence. Cette absorption a pour effet de faire disparaitre la classe ouvrière des sciences sociales d'abord et du langage ensuite, troublant la compréhension des rapports sociaux.

Dès 1968, H. Lefebvre parlait des classes moyennes comme d'une mystification terminologique et citait la presse en mettant valeurs, culture et supérieures entre guillemets (je les mets en italiques) :

«Une nouvelle mystification monte: les classes moyennes n'auront qu'une ombre de pouvoir, que des miettes de richesse, mais c'est autour d'elles que s'organise le 
scénario. Leurs valeurs, leur culture l'emportent ou semblent l'emporter parce que supérieures à celles de la classe ouvrière » (Lefebvre, 1968 : 82). cotisations sociales sont définies comme une part du salaire qui rémunère un travail, ce champ sémantique est reformulé du point de vue des intérêts opposés du patronat qui suggère que ces cotisations sont retranchées du résultat de l'activité de l'entreprise. Dans les termes techniques de la philologie, on dira que l'isotopie du poids (« charge : Ce qui pèse ", Grand Robert, éd. 2001, entrée charge) permet de filer la métaphore grâce à une suite de pas rhétoriques : remplacer cotisations sociales par charges sociales permet de dire qu'elles pèsent, qu'elles sont lourdes (synonyme), trop lourdes (superlatif), qu'il faut les alléger (antonymie). C'est de la même façon qu'un plan de licenciement est renommé plan social, le mot social pris dans ce contexte inversant son sens de «protection des salariés » à « exclusion de l'emploi ». dire la chose) est toujours là, et comment classe ouvrière en italique (c'est-à-dire le mot) a disparu, l'absence du mot permettant de ne plus parler de la chose par un effet de censure invisible. quantitativement et non plus qualitativement, par exemple à partir des statistiques de la Direction générale des Impôts. En 2011 (chiffres arrondis) :

a. 0,3 million (moins de $1 \%$ des ménages identifiés par un taux marginal d'imposition de $41 \%$ ) payait 15,3 Mds $€$ ( $30 \%$ de l'impôt sur le revenu des personnes physiques - IRPP), catégorie de la population assimilable à la « bourgeoisie » au sens historique du mot ;

b. 17 millions de ménages ( $45 \%$ des déclarants) payaient $37 \mathrm{Mds} €$ ( $70 \%$ de l'IRPP), constituant la « classe moyenne » (au singulier) en tant que distincte de la catégorie « la plus aisée » de la société d'un côté et des « classes populaires » de l'autre ;

c. 20 millions de ménages déclarant des revenus ( $54 \%$ du total des foyers fiscaux) étaient non imposables, constituant les « classes populaires ».

En dehors d'une couche sociale très déclassée dite «du quart monde » qui ne déclare pas de revenus et dont la catégorie est difficile à cerner, le critère du revenu permet de distinguer trois classes :

a. une classe supérieure fortunée constituée par environ $1 \%$ des ménages payant en moyenne $50000 €$ d'IRPP par an ; 
b. une classe moyenne stricto sensu (au singulier) constituée par $45 \%$ des ménages, imposés à hauteur de $2200 €$ en moyenne ;

c. une troisième catégorie, $54 \%$ des ménages, non imposés au titre de l'IRPP, qui constitue ce qu'on appelait naguère « les classes laborieuses », voire « le prolétariat », salariés agricoles et de l'industrie mais aussi employés des services ${ }^{4}$.

Comme on va le voir dans la section suivante, l'expression classes moyennes (au pluriel) inclut désormais cette dernière catégorie. En fait, elle couvre comme une classe unique la totalité du champ social à l'exception de ses extrémités qui ne comptent que quelques pourcents de la population : personnes désocialisées d'un côté et grandes fortunes de l'autre ${ }^{5}$.

Dans Les Classes moyennes - volume n³ 982 de la collection «Que sais-je ? » paru en 2013 -, Julien Damon, spécialiste incontesté du sujet, compte moins de 5 dépêches AFP par an titrées en « classes moyennes » avant 2000, 15 en 2006, 30 en 2009, plus de 60 en 2012 - une progression logarithmique. La consultation en ligne des quotidiens Le Monde, Le Figaro, Libération, Les Échos ou La Tribune, celle des hebdomadaires Marianne, Le Point, L'Express et Le Nouvel Observateur fait apparaitre un emploi quotidien de «classes moyennes ", à la fois objet d'enquête et sujet de l'Histoire.

Les exemples qui suivent (section 2) montrent que la confusion entre les classes moyennes et la population en général pénètre le corps social et l'«opinion publique » avec toute la puissance du fait accompli ou des choses comme elles sont. Pour ce qu'il est désormais convenu d'appeler une « sociologie de presse » (Chauvel, 2014), il n'existe plus de lutte des classes puisqu'il n'existe plus de classes, mais uniquement des situations sociales individuelles ou catégorielles mal définies, déconstruites, et finalement anomiques.

Cette situation pose au linguiste deux questions liées : 1) la première est de comprendre comment s'est produite cette absorption improbable, sachant que dans le système terminologique remis en cause, les notions de "classe ouvrière " et de "classe moyenne » se définissaient par leur incompatibilité ; 2) la seconde est de comprendre comment les mots peuvent à ce point non pas valoir pour les choses, mais tromper à leur sujet.

\section{2. «Classes moyennes » et « classe moyenne »}

Les occurrences de classe moyenne et classes moyennes relevées pour illustrer le phénomène entre le 8 juillet et le 20 août 2014 sur les sites du Monde et du Figaro montrent bien la disparition totale, dans la presse française, du champ sémantique de la classe ouvrière, $\mathrm{du}$ salariat et $\mathrm{du}$ prolétariat, au profit de synonymes et parasynonymes relevant de la « sociologie de presse ».

6 Il est important de noter que toutes les occurrences relevées entre ces deux dates sont citées ci-dessous; seuls les doublons (dans un même quotidien ou d'un quotidien à l'autre) n'ont pas été retenus.

Ces occurrences mettent à jour une opposition significative entre classe moyenne au singulier et classes moyennes au pluriel, l'arrivée d'une "nouvelle classe moyenne » au singulier dans les pays émergents succédant à l'expansion des «classes moyennes traditionnelles » au pluriel dans les pays avancés. 


\section{l'été 2014 s'est centrée en France sur la préparation de la loi de finances 2015 et sur} l'IRPP :

(1) «Le premier ministre a annoncé que la loi de finances 2015 prévoirait une baisse d'impôt sur le revenu pour les classes moyennes " (Le Figaro, 08/07/14, "Baisse d'impôt pour les classes moyennes en $2015 »)$.

(2) «La volonté de Manuel Valls de faire un geste en direction des classes moyennes dans le cadre du budget...» (Le Figaro, 12/08/14, «Impayés fiscaux : quand trop d'impôt tue l'impôt »).

Dans (1) et (2), les classes moyennes vont de soi ; elles désignent les catégories sociales auxquelles les lecteurs du journal s'identifient, en quelque sorte «tout le monde»; elles sont définies comme payant l'impôt - mais excluent les plus grandes fortunes, lesquelles ne bénéficieront pas d'une " baisse d'impôt ».

Le Monde - exemples (3) à (6) - utilise lui aussi classes moyennes au sens de « contribuables » et cible son lectorat, avide d'informations sur le sort qui l'attend:

(3) «Les classes moyennes ont connu des hausses parfois considérables [de l'impôt] que personne ne sait leur expliquer» (Le Monde, 12/08/14, « Pas de réformes sans principes »).

(4) « Dans le prochain projet de loi de finances [...], le gouvernement devrait à la fois pérenniser ces mesures de réduction d'impôt pour le bas du barème et, même, les amplifier pour en faire bénéficier les classes moyennes » (Le Monde, 20/08/14, « Moins d'un foyer fiscal sur deux paiera l'impôt sur le revenu en $2014 »)$.

(5) «La mobilisation de 5 milliards d'euros au service de la mise en œuvre d'un barème progressif permettrait de diminuer les prélèvements sur les moins favorisés sans augmenter ceux qui pèsent sur les classes moyennes» (Le Monde, 15/08/14, « Non, la décision du Conseil constitutionnel n'interdit pas la CSG progressive »).

(6) «Dans le prochain projet de loi de finances, cette enveloppe devrait être portée à 2 milliards d'euros [...] afin de faire bénéficier les ménages des classes moyennes inférieures d'une baisse de l'impôt sur le revenu» (Le Monde, 18/08/14, «Les six dossiers brûlants qui menacent d'empoisonner l'élysée à l'automne »).

L'exemple (4) montre une stratification entre les classes moyennes et le bas du barème, expression qui désigne les ménages d'ouvriers et d'employés qui en sont exclus (ils finiront d'ailleurs non imposables). Dans l'exemple (5), ils sont nommés les moins favorisés, masculin pluriel recouvrant non pas une classe ni même une catégorie, mais un ensemble de situations individuelles. Cependant, dans l'exemple (6), les mêmes sont réintégrés dans les classes moyennes dites inférieures.

(7) «Reste que cette fusion [prime pour l'emploi (PPE) + revenu de solidarité active (RSA)] ferait beaucoup de "perdants", notamment les ménages aux revenus moyens qui touchaient la prime pour l'emploi grâce aux règles s'appliquant aux concubins et prenant en compte le nombre de personnes à charge. Bref, des classes moyennes dont Manuel Valls veut justement baisser la fiscalité en 2015. [...] Reste que la baisse de l'impôt sur le revenu [...] devra intégrer non seulement la pérennisation de la mesure en direction des ménages modestes votée cet été mais aussi recycler tout ou partie des 2,5 milliards d'euros de ristourne de cotisations salariales retoqués par le Conseil constitutionnel début août. Sans compter que le gouvernement a promis, en plus, de baisser en 2015 les impôts des classes moyennes» (Le Figaro, 18/08/14, «Baisses d'impôts : les trois pistes de l'exécutif »).

En (7), Le Figaro associe les ménages modestes aux classes moyennes après avoir expliqué que les ménages aux revenus moyens peuvent bénéficier de la prime pour l'emploi, alors qu'on sait que celle-ci est allouée sous des conditions de ressources qui font de ses bénéficiaires les salariés les moins aisés ${ }^{6}$. Tout se passe ici comme si le 
premier critère d'appartenance aux classes moyennes était d'avoir un emploi, y compris au Smic et à temps partiel, ce qui inclut les travailleurs pauvres. D'où l'exemple (8) où les classes moyennes descendent jusque dans le tiers de la population française qui n'a pas les moyens d'envoyer ses enfants en vacances :

(8) «Cet été, même les classes moyennes affluent au service jeunesse, qu'elles fréquentaient peu. Employés, fonctionnaires, cadres moyens expliquent privilégier les activités de leurs enfants à prix raisonnables » (Le Monde, 12/08/14, « Sacrifier ses vacances, cet autre effet de la crise »).

Cet exemple (8) confirme l'assimilation des catégories non imposables au même continuum social que les cadres moyens. La paupérisation d'une partie de ces classes moyennes nouvelle manière sous l'effet de la crise n'empêche pas l'extension néolibérale du concept de continuer à absorber les catégories dites « de la France d'en bas » :

(9) «[Le gouvernement vient] de prendre conscience du prix politique du "matraquage fiscal" de classes moyennes à qui on avait juré, la main sur le cœur... » (Le Monde, 09/08/14, « Il faut réformer la CSG pour soulager les bas revenus »).

C'est aussi ce qui apparait dans l'exemple (10), extrait d'une interview de J. Damon, auteur du « Que sais-je ?» déjà cité, sociologue professeur à Sciences Po :

(10) «[...] les Français, et parmi eux aux premiers rangs les classes moyennes, sont extrêmement pessimistes. [...] Certes les très très riches (disons moins de $1 \%$ de la population) sont de plus en plus riches. Certes le nombre de pauvres (au sens monétaire traditionnellement utilisé en France et en Europe) est en croissance, mais je réfute l'idée selon laquelle les pauvres sont de plus en plus pauvres. [...] Les classes moyennes ont le profond sentiment de se paupériser, ce qui est très discutable également. Mais on peut faire simple et efficace : il est vrai que les classes moyennes inférieures (surtout des salariés privés et des indépendants) se prennent les chocs $\mathrm{du}$ chômage et donc voient leurs revenus baisser. Il y a incontestablement un rapprochement des situations des classes moyennes inférieures de celles des plus défavorisés. [...] Les États-Unis [...] voient [...] la dualisation notable de leur population entre une classe aisée et une classe défavorisée qui apporte aux mieux dotés les services dont ils ont besoin. [...] On aura toujours besoin de dirigeants et d' exécutants, mais pas forcément des catégories intermédiaires... » (Le Figaro, 02/07/14, «Pauvres plus pauvres et riches plus riches: où sont passées les classes moyennes?» à propos d'un rapport de l'Insee pointant l'aggravation des inégalités).

Chercheur patenté, salué par ses pairs (voir Chauvel, 2014), J. Damon rejoint ici la sociologie de presse, décrivant une société française où les classes ne sont pas antagonistes mais complémentaires, comme dans l'apologue des membres et de l'estomac ${ }^{7}$. Il commence par opposer aux classes moyennes - dont on vient de voir qu'elle n'est pas une classe, mais plusieurs, comme le pluriel l'indique - les «très très riches » et les pauvres en tant qu'individus. Il distingue ensuite les « classes moyennes inférieures » (elles aussi multiples) des plus « défavorisés » (au masculin pluriel, dénote ici encore des individus), puis deux pôles spécifiques résultant de la division critique des «classes moyennes» entre "classe aisée» (les «mieux dotés») et «classe défavorisée ", ou encore entre "dirigeants» et "exécutants» tandis que les "catégories intermédiaires» sont appelées à disparaitre. Cette division des classes moyennes redualise la société sur des bases nouvelles où la classe laborieuse n'est plus paysanne ou ouvrière, mais de services (à la personne) (Gorz, 1983).

Du côté des BRICS (Brésil, Russie, Inde, Chine, Afrique du Sud) et autres pays en développement, on voit apparaitre un autre implicite significatif : une classe moyenne au 
singulier émerge comme par génération spontanée de classes laborieuses qui restent innommées :

(11) « [Afrique] ... la Chine notamment, cherche depuis plusieurs années à s'y placer en première ligne, pour répondre à la demande d'une classe moyenne qui représentera dans les prochaines années entre 300 et 500 millions de consommateurs, a souligné le ministre français des Finances Michel Sapin... » (Le Figaro, 15/07/14, «Création d'une fondation franco-africaine »).

(12) « [Chine] D'autre part, faire émerger une classe moyenne permettant d'accroître la demande intérieure. En Chine, la consommation domestique a compté pour plus de la moitié du PIB au cours du premier semestre... » (Le Monde, 06/08/14, «La Chine relève le salaire minimum pour dynamiser la consommation »).

(13) «[Iran] Cette dernière opération militaire israélienne a suscité une vague inédite de solidarité avec les Palestiniens parmi des Iraniens issus majoritairement de la classe moyenne, éduqués et connectés à Internet... » (Le Monde, 11/08/14, «Les opposants iraniens affichent désormais leur solidarité avec les Palestiniens »).

(14) « [Turquie] Une politique forte du service public a élargi le terrain d'action de la classe moyenne du secteur de la santé au secteur des transports et de la technologie, tout en augmentant la qualité de vie et le climat de confiance» (Le Monde, 11/08/14, «Erdogan, une "machine" à gagner les élections »).

(15) «[Corée du Sud] Tout semblait réussir: technologies vertes, villes intelligentes... Mais la "démocratie économique" n'était pas au rendez-vous; la cohésion sociale s'effritait et la classe moyenne perdait ses illusions sur les bienfaits de l'expansion » (Le Monde, 13/08/14, «Le pape François va découvrir une Corée du Sud en plein doute »).

(16) «[Russie] La nouvelle classe moyenne supérieure, habituée à acheter des fromages français, des glaces italiennes, des biscuits belges ou de la viande australienne dans de luxueux supermarchés, devra s'adapter » (Le Monde, 12/08/14, « La popularité de Vladimir Poutine à l'épreuve de l'embargo russe »).

(17) «[Chine] ... l'avènement de la classe moyenne supérieure chinoise comme force financière sur les marchés occidentaux, boostée par un taux de change du dollar favorable au yuan, capable de payer entièrement en cash, et particulièrement attirée par Los Angeles, San Francisco, Las Vegas, Seattle et Manhattan...» (Le Figaro, 30/07/14, «États-Unis: les riches Chinois dopent un marché immobilier convalescent »).

37 L'opposition entre d'un côté les classes moyennes (au pluriel, exemples 1 à 10) des pays avancés et de l'autre la classe moyenne (y compris supérieure, au singulier, exemples 11 à 17) des pays émergents s'explique aisément : pour la sociologie de presse, il n'existe de classe ouvrière et de prolétariat rural que dans les pays nouvellement industrialisés : ces pays sont en développement parce qu'ils ont des prolétariats ruraux et ouvriers. Il n'y a donc une classe moyenne sui generis que dans les pays où existent aussi une classe ouvrière et une paysannerie.

Le recentrage des économies développées sur les services dont les emplois sont considérés comme relevant d'un statut social de type moyen (comme si tertiaire signifiait intermédiaire) permet de définir les pays les plus avancés comme des populations de classes moyennes. Une exception à cette opposition systématique entre singulier et pluriel vient dans l'exemple qui suit :

(18) «"Le pouvoir d'achat des classes moyennes n'a cessé de baisser depuis deux ans", a indiqué la numéro un du syndicat des cadres lors d'une conférence de presse. [...] "La CFE-CGC [Confédération française de l'encadrement-Confédération générale des cadres] réclame plusieurs mesures en faveur de la classe moyenne, dont la baisse de la fiscalité sur la participation et l'intéressement et le retour du forfait social à $8 \%$ au lieu de $20 \%$ » (Le Figaro, 03/07/14, « Conférence sociale : CFE-CGC participe »). 
Pour Carole Couvert, présidente de la CFE-CGC, syndicat voué à la défense des intérêts des cadres, ceux-ci sont les seuls à constituer «la classe moyenne » authentique, au singulier, c'est-à-dire au sens où l'entend la sociologie historique ${ }^{8}$.

Je citerai J. Lojkine (2012) pour conclure à ma place ce premier examen :

«La classe moyenne, c'est l'anticlasse, la classe qui ne s'oppose à aucune autre parce qu'elle est censée les absorber toutes. [...] En période de crise, la classe moyenne devient synonyme de société des "inclus" opposés aux "exclus", substitut du clivage de classe : la classe moyenne devient alors le porte-drapeau des salariés à statut enfermés dans leurs ghettos de "riches", entourés par la masse anonyme des "sans" : sans-papiers, sans-travail, sans-domicile, sans-patrie. »

Et plus loin :

«La crise [...] a fait éclater ce faux concept en révélant les formes nouvelles des rapports de classes. La "classe moyenne" cache en réalité un conflit majeur entre des fractions prolétarisées du salariat intellectuel et une fraction ultraminoritaire des cadres dirigeants [...]. Chômage, précarisation, paupérisation [...] dessinent des formes nouvelles de prolétarisation [qui] pour autant [ne s'identifient pas] au prolétariat ouvrier.»

\section{Retour sur classe ouvrière}

Les frontières entre classes ou catégories - personnes déclassées $\leftrightarrow$ classes moyennes $\leftrightarrow$ grandes fortunes - ne sont ni tranchées ni étanches, les classes moyennes pouvant même se définir par des critères psychologiques tels la peur du déclassement et le désir d'ascension.

Que ce soit au singulier ou au pluriel, le syntagme classe(s) moyenne(s) conserve avec le morphème classe(s) ce qu'il faut pour recouvrir le classe de classe ouvrière ou le classes de classes laborieuses, avant de les absorber - la classe ouvrière, quant à elle, existant en sociologie pré-Chicago non pas en tant que classe unique, mais en tant que classe singulière, porteuse de l'émancipation de l'humanité.

Désormais chargée de désigner la quasi-totalité de la société française, l'expression classes moyennes fonctionne comme un équivalent de population. Pas plus que classe ouvrière (pour le singulier) ou classes laborieuses, classes populaires (pour le pluriel), la sociologie de presse n'emploie classes pauvres ou classes riches. Le mot moyen lui-même, qui signifie en principe "entre deux choses », " intermédiaire » (Grand Robert, entrée moyen) n'a plus cette valeur dans ce cotexte. Le morphème inférieur étant évincé des discours «politiquement corrects » (de même que la Charente inférieure, en 1941, la Seine inférieure, en 1955, la Loire inférieure, en 1957, et les Basses Pyrénées, en 1969, sont devenues maritimes et atlantiques) un grand nombre de termes nouveaux comme SDF (sans domicile fixe), RMI (revenu minimum d'insertion), RSA, quart monde, temps partiels, demandeurs d'emploi, chômeurs en fin de droits, travailleurs précaires, travailleurs intérimaires, CDD (contrats à durée déterminée) sont apparus pour les désigner. La multiplication de ces termes contraste avec l'unité d'emploi de classes moyennes; elle apparait comme un symptôme du défaut de vérité de la sociologie de Chicago.

L'expression classe(s) moyenne(s) ne s'oppose plus qu'à classes supérieures, classes aisées, assez aisées, avec les variantes moins aisées et plus aisées. Corollairement, bourgeoisie, grande bourgeoisie et aristocratie, noblesse ne sont plus considérées comme des classes 
mais comme des situations dynastiques ou familiales, sinon individuelles. Le centre de gravité des classes moyennes est ainsi tiré vers le bas9.

La terminologie des classes moyennes (au pluriel) est d'origine anglo-saxonne et antérieure à K. Marx. Le tout premier sens historique de classe (du latin classique classis, "catégorie de citoyens ») est celui de « regroupement de personnes ayant en commun une fonction, un genre de vie, une idéologie, etc. » (Grand Robert, entrée classe). Le sens dérivé très voisin mais plus spécialisé de "communauté d'intérêts économiques et politiques " est apparu au cours des luttes politiques de la Révolution française (1788-1791), notamment dans l'expression classe sociale (ibid.). Mais le type de société dans lequel la notion de "classe » s'est développée prend sa source dans la révolution industrielle anglaise (à partir de 1750) qui a progressivement opéré la jonction de l'aristocratie terrienne, hereditary upper class, et de la grande bourgeoisie entrepreneuriale urbaine, upper middle class, terme opposé à middle class en général et à lower middle class en particulier. Dépassant l'approche marxiste qui oppose fondamentalement prolétariat et bourgeoisie par la prise en compte de facteurs structurels, M. Weber (par exemple dans Économie et Société, posthume, 1921) a défini l' upper middle class britannique par un critère culturel - niveau supérieur d'éducation: façon de parler, cursus scolaire et universitaire - qu'elle partage aujourd'hui avec la gentry traditionnelle. Tout en bas de l'échelle, on trouve les labouring classes (nos classes laborieuses). Dans cette conjoncture, la coupure constitutive de l'ordre social se situe entre la middle class des owners (les possédants, propriétaires et rentiers) et la working class des workers (les travailleurs, non possédants), entre bourgeoisie d'un côté (grande et petite confondues) et prolétariat de l'autre (dont les membres sont quant à eux toujours susceptibles de tomber dans le sous-prolétariat). Au départ, les classes moyennes (middle classes) ne sont pas définies comme travailleuses pour des raisons essentiellement historiques, le Royaume-Uni ayant longtemps bénéficié de l'expansion continue de son empire colonial.

Désormais sortie de ce cadre, la définition des classes moyennes suscite de nombreux débats (Chauvel, 2014). Pour les uns, la définition est statutaire: "Le niveau d'éducation, le statut socio-économique de l'emploi, le mode de consommation et un ensemble particulier de valeurs concourent à caractériser le groupe. » Pour les autres, la classe moyenne regroupe les individus dont le revenu est situé entre 75 et $125 \%$ de la médiane. Pour d'autres encore l'appartenance à la classe moyenne relève de l'autoidentification des individus mais cette dernière définition pose problème dans la mesure où $62 \%$ des Maliens se voient comme membres des classes moyennes contre seulement $40 \%$ des Polonais, pourtant huit fois plus riches et dont les revenus sont nettement plus resserrés (voir aussi Quénot-Suarez, 2013).

Selon S. Pressman (2007) enfin, la classe moyenne n'est pas définie par ses limites mais par son centre, à partir duquel différents critères permettent les comparaisons dans le temps et dans l'espace.

Si la notion de "prolétariat » (littéralement, « la classe qui n'a pas d'autre richesse que les enfants »), qui renvoie à la grande misère de la classe ouvrière du xixe siècle, peut être considérée comme obsolète en France, la "misère du monde", comme disait P. Bourdieu, est toujours là. Le fait que la classe ouvrière et les catégories assimilées soient désormais recouvertes par les classes moyennes et autres catégories intermédiaires placées entre les "laissés pour compte » et les "maitres du monde » constitue une tromperie délibérée, même si la division majeure semble aujourd'hui 
davantage d'ordre culturel et social qu'économique, car tous trois vont de pair et se surdéterminent mutuellement.

Certes, la composition de la société, en France, a beaucoup changé depuis le début ou même le milieu du XXe siècle, et avec elle la stratification des statuts : la paysannerie a pratiquement disparu et le salariat industriel, victime de la redistribution mondiale du travail, suit la même voie. Le développement des services et la féminisation du travail ont partiellement compensé la désindustrialisation de l'activité, entrainant l'augmentation du nombre de «travailleurs pauvres» et «précaires» dont le destin (gagner moins qu'il n'est nécessaire pour vivre et élever dignement ses enfants) est tout à fait comparable à celui du prolétariat d'autrefois.

51 On ne peut pas ne pas citer à ce sujet le rapport du député socialiste O. Ferrand (président de Terra Nova, décédé le 30 juin 2012) prônant en substance l'abandon des classes populaires par le Parti socialiste ${ }^{10}$. La synthèse de ce rapport commence par décrire la France d'hier et d'aujourd'hui :

«La coalition historique qui a porté la gauche depuis près d'un siècle, fondée sur la classe ouvrière, est en déclin. C'est vrai en France, comme dans le reste de l'Europe et aux États-Unis. Depuis le Front populaire en 1936, la gauche en France (socialiste, mais surtout communiste) a accompagné la montée en puissance du monde ouvrier. La victoire de François Mitterrand à l'élection présidentielle de 1981 leur est intimement liée : la classe ouvrière est à son apogée démographique (37\% de la population active) et vote massivement à gauche [...]. Autour de ce cœur ouvrier s'est constituée une coalition de classe : les classes populaires (ouvriers, employés) et les catégories intermédiaires (les cadres moyens). »

Ce discours reprend la terminologie de l'époque prétendument révolue où elle avait cours : classe ouvrière, monde ouvrier, classe ouvrière de nouveau, cœur ouvrier, coalition de classe, classes populaires. Puis vient l'amorce de ce qui va prendre la relève : catégories (et non plus classes) intermédiaires et cadres moyens.

La suite décrit le « rétrécissement démographique de la classe ouvrière » qui tombe à $13 \%$ dans l'industrie, accompagnant l'effondrement (moins $40 \%$ ) du « socle électoral » de la gauche, phénomène amplifié par la dévitalisation du sentiment de classe en raison de la recomposition du monde du travail au profit des ouvriers qualifiés et des salariés du tertiaire « qui se reconnaissent davantage dans les classes moyennes » où le rapport au travail est de type individualiste et gomme la conscience de classe.

«Comment la classe ouvrière a-t-elle pu disparaître depuis les années Mitterrand ? »se demandent 0 . Ferrand et al. (ibid.). La question présuppose cette disparition, et l'explication qu'ils en donnent est d'ordre culturel : la génération de 1968 a entrainé la gauche vers les questions de société, la liberté sexuelle, la contraception, l'avortement, l'émancipation des femmes, la tolérance, l'ouverture aux différences, une attitude favorable aux immigrés, à l'islam, à l'homosexualité, la solidarité avec les démunis. Les ouvriers ont fait le chemin inverse en adhérant aux valeurs traditionnelles "petitebourgeoises». La rupture aurait été accentuée par la «tertiarisation» de l'économie qui a entrainé le déclin de la classe ouvrière de pair avec la précarisation du travail, la perte de l'identité collective, de la solidarité et de la fierté de classe, la relégation dans les quartiers, créant des réactions contre les immigrés, les assistés, la perte des valeurs morales et les désordres de la société contemporaine :

«Les déterminants économiques perdent de leur prégnance et ce sont les déterminants culturels, renforcés par la crise économique, "hystérisés" par 
l'extrême droite, qui [...] expliquent le basculement vers le Front national et la droite $»^{11}$. prolétarienne ayant été remplacés depuis la fin des Trente Glorieuses (à partir de 1973-1981 donc) en nombre à peu près égal par des caristes et des camionneurs isolés dans leur emploi, individualistes ou du moins beaucoup moins pourvus de « conscience de classe ». Quant à l'expression classes populaires, elle se maintient selon lui dans la mesure où elle permet de continuer à rapprocher les ouvriers et les employés, tant en termes de revenus qu'en ce qui concerne le statut social, le chômage, l'insécurité et la précarité de l'emploi (CDD et contrats d'intérim représentant désormais $90 \%$ des nouveaux contrats de travail, voir Le Figaro du 25/07/14). Il note aussi que la « classe ouvrière » de référence était plutôt constituée de professionnels qualifiés que d'ouvriers spécialisés immigrés. 


\section{Vers des luttes sociales à base ethnique}

\section{Comment des mots peuvent-ils tromper
poursuivant la réflexion de V. Klemperer,}

« il n'y a pas de doute que les mots avec lesquels on a beaucoup menti sont devenus eux-mêmes peu fiables. Que l'on essaie seulement de prononcer des mots comme [...] Lebensraum (espace vital) ou Endlösung (solution finale), la bouche elle-même s'y refuse [...]. Celui qui malgré tout s'en sert est soit un menteur soit une victime du mensonge. Les mensonges pervertissent le langage. Et il n'existe aucune thérapie pour les mots corrompus; on est obligé de les expulser du langage. Plus c'est rapidement et radicalement fait, mieux ça vaut $\%$.

61

L'absorption de classe ouvrière par classes moyennes n'est pas le seul mensonge de la sociologie de Chicago. É. Fassin (2014, cité d'après les bonnes feuilles parues dans Mediapart le 07/05/14) note :

«Depuis les années quatre-vingt, [...] les majorités républicaines des deux bords prétendent s'attaquer, non à l'immigration [...], mais au problème qu'elle représenterait. On affirme ainsi éviter la xénophobie, voire lui répondre. En réalité, c'est reprendre à son compte [...] une construction qui constitue l'immigration en problème, et ainsi pose les conditions de la xénophobie politique au motif de la combattre.»

Et plus loin :

«Un déplacement s'est opéré à partir des années 1980. Sans doute le mot intégration apparait-il moins contraignant qu'assimilation. Toutefois, derrière le substantif, le verbe a changé sans qu'on en prenne vraiment conscience : intégrer a cédé la place à s'intégrer [et la pronominalisation] fait basculer la responsabilité de l'intégration de la société à l'immigré [...]. Ainsi, l'intégration aussi devient un problème insoluble. »

Plusieurs thèses et ouvrages et de nombreux articles parus dans la revue Mots et dans Langage et société ont montré la "négativisation " progressive de la question immigrée et comment au tournant des années 1980, on est passé, dans la presse de gauche comme de droite, d'un cadrage sur les problèmes vécus par les immigrés (logement, droits, alphabétisation) aux problèmes posés par les immigrés (délinquance, baisse de niveau dans les écoles, terrorisme) (Bonnafous, 1999). S. Bonnafous, elle-même pionnière des recherches en lexicométrie diachronique, a soutenu en 1990 une thèse d'État publiée en 1991 sur le discours sur les immigrés et sur l'immigration dans la presse française de 1974 à 1984. J. Guilhaumou (1992) en résume ainsi les conclusions :

«L'étude du temps lexical, de la variation chronologique des formes [de travailleurs immigrés à immigrés, à immigrés clandestins, à immigrés sans papiers], souligne les moments forts d'une telle déshérence des représentations de type classiste au sens large : le tournant des années 1979-1980 et l'amplification des années 1983-1984 [dû au "tournant de la rigueur"]. Si en 1974, le point de vue adopté [par la presse] est celui des immigrés eux-mêmes, soucieux de leurs droits, en 1984, il n'est plus question que des "Français" et de leurs privilèges à sauvegarder. Le "complexe de l'immigration" rend compte de l'introduction progressive de la vision extrême dans l'opinion publique.»

La réduction à gauche du syntagme travailleurs immigrés par la suppression de travailleurs et son extension à droite par l'adjonction de clandestins transforme l'adjectif en substantif; immigré cesse de désigner une situation pour dénoter une essence. Le glissement sémantique parti du signifiant cible le signifié, les immigrés eux-mêmes, et leurs enfants, désormais indésirables. Un dernier glissement enclôt les «minorités 
visibles » telles que sous-représentées dans la fiction (cinéma, téléfilms, séries télévisées), mais surreprésentées dans les actualités et les faits divers, synonymes par collocations récurrentes de problème, complication, délinquance, menace, alerte, danger et violence :

«La fiction française, notamment, reste singulièrement dominée par la classe moyenne blanche (comme la fiction américaine des années cinquante) [...] On s'interroge sur la difficulté des scénaristes français à intégrer (au sens le plus simple du mot) dans leurs récits des personnes d'origine étrangère [...] en montrant simplement leur vie quotidienne " (Bourdon, 1993, je souligne).

Dans les années qui ont suivi, notamment à partir des années 2000, le thème de la classe moyenne blanche, a pris le relais, thème qui recycle la racialisation des rapports de classe amorcée par la substitution de travailleurs par immigrés. C'est pratiquement dans le même mouvement néologique qui voit classes moyennes absorber classe ouvrière et où la classe ouvrière elle-même "disparait» (les guillemets sont importants) que les mouvements sociaux les plus durs ont acquis en France des bases ethniques en substitution des bases de classe.

\section{Conclusion}

Pour conclure, on ne peut qu'être frappé par l'aspect unanime des glissements sémantiques opérés par le discours des médias. Comme si les journalistes s'étaient « donné le mot». Ou comme si la contrefaçon terminologique avait envahi le marché linguistique au point de remplacer les mots justes par des copies, par des mots faux. Comme disait Thomas Gresham, "la mauvaise monnaie chasse la bonne ». Mais qui peut se vanter de posséder les "bons mots", les «mots justes»? Et quelle est la moralité, ou la morale, de cette histoire ? En fait, il n'y en a pas, du moins pas au plan linguistique, même s'il y en a une au plan politique. Le plan linguistique - langagier ou sémiotique - n'est pas politiquement neutre, mais divisé par les intérêts de classe. En particulier, la terminologie du champ social est traversée par un conflit opposant les intérêts contradictoires qui sont à la fois la cause et l'effet de mutations sociales survenant dans la distribution et l'organisation du travail. Longtemps protégées de la rapacité du capital par le programme du Conseil national de la Résistance, les classes populaires et les classes moyennes françaises voient aujourd'hui leur sort aligné sur la moyenne des situations qui leur sont faites dans les pays les plus avancés. Dans cette conjoncture, la réalité s'appuie idéologiquement sur le langage pour, sans considération de moralité, ni d'immoralité non plus, faire évoluer les choses dans le sens des intérêts dominants. Comme l'a dit Warren Buffett (voir l'épigraphe de cet article), « il y a une lutte des classes, c'est sûr, et c'est ma classe, la classe des riches, qui fait cette guerre, et c'est nous qui sommes en train de la gagner ». C'est à voir. Demain n'est pas écrit. 


\section{BIBLIOGRAPHIE}

BONNAFOUS, S. (1991) : L'Immigration prise aux mots. Les immigrés dans la presse au tournant des années 80, Paris, Kimé.

- 1999, «La médiatisation de la question immigrée : état des recherches », Études de communication, 22, «La médiatisation des problèmes publics ».

BOURDON, J. (1993) : « Les étrangers au prime time ou la télévision est-elle xénophobe ? ", in : C. Frachon \& M. Vargaftig (dirs), Télévisions d'Europe et immigration, Paris, Ina-Association Dialogue entre les cultures, p. 31-42.

BURAWOY, M. (2014 [2012]) : « La domination culturelle : quand Gramsci rencontre Bourdieu », trad. de l'anglais, Contretemps (en ligne : Conversations with Bourdieu : The Johannesburg Moment, consulté le 15/08/14).

CAHEZ, P. (2013) : « Le mythe de la classe moyenne », Médiapart, 15 aout.

CHAUVEL, L. (2014) : « Moyennisation ou polarisation ? La dynamique des classes moyennes en France et dans un monde globalisé », Cahiers français, 378, p. 21-27.

DA CUNHA, D. (1992) : Discours rapporté et circulation de la parole, Louvain-la-Neuve, Peeters (coll. « Bibliothèque des cahiers de l'Institut de linguistique de Louvain »).

DAMON, J. (2013) : Les Classes moyennes, Paris, Presses universitaires de France (coll. « Que saisje?»).

Delhommais, P.-A. (2013) : «L'été des classes moyennes », Le Point, 25 juil. (en ligne : http:// www.lepoint.fr/editos-du-point/pierre-antoine-delhommais/l-ete-des-classesmoyennes-25-07-2013-1708110_493.php, consulté le 15/08/14).

FASSIN, É. (2014) : Gauche, l'avenir d'une désillusion, Paris, Éd. Textuel.

FERRAND, O., JEANBART, B. \& PRUDENT, R. (2011) : Gauche : quelle majorité électorale pour 2012 ?, rapport, Paris, Terra Nova (en ligne : http://www.tnova.fr/sites/default/files/ Rapport\%20Terra\%20Nova\%20Strat\%C3\%A9gie\%20\%C3\%A9lectorale.pdf, consulté le 15/08/14). GORZ, A. (1983 [1980]) : Adieux au prolétariat, rééd. augmentée, Paris, Éd. Le Seuil. GRAEBER, D. (2014) : Comme si nous étions déjà libres, Montréal, Lux Éd. GUILHAUMOU, J. (1992) : « Simone BONNAFOUS, L'Immigration prise aux mots. Les immigrés dans la presse au tournant des années $80 »$, Histoire \& Mesure, 3, vol. 7, p. 349-350.

JACOBY, R. (2014) : «Thomas Piketty ou le pari d'un capitalisme à visage humain », Le Monde diplomatique, 725 , aout.

KLEMPERER, V. (1996) : Lingua Tertii Imperii, la langue du Troisième Reich, Paris, A. Michel.

LEFEBVRE, H. (1968) : La Vie quotidienne dans le monde moderne, Paris, Gallimard.

LOJKINE, J. (2005) : Adieu à la classe moyenne ?, Paris, Éd. La Dispute.

- (2012) : « La classe moyenne, cette anticlasse censée les absorber toutes », L'Humanité, 18 janv.

MISCHI, J. (2014) : Le Communisme désarmé. Le PCF et les classes populaires depuis les années 1970, Marseille, Agone.

PAVEAU, M.-A. (2013) : Langage et Morale. Une éthique des vertus discursives, Limoges, Lambert-Lucas. 
PRESSMAN, S. (2007) : «The Decline of the Middle Class : An International Perspective », Journal of Economic Issues, vol. 41, p. 181-200.

QUÉNOT-SUAREZ, H. (2013) : Alternatives internationales, hors-série, « L'Afrique qui bouge » (en ligne : http://www.alternatives-internationales.fr/les-classes-moyennes-laramenent_fr_art_1216_63860.html, consulté le 15/08/14).

ROTHÉ, B. (2013b) : De l'abandon au mépris. Comment le PS a tourné le dos à la classe ouvrière, Paris, Éd. Le Seuil.

- (2013b) : «PS et ouvriers : “On est passé de l'abandon au mépris” », interview par Dominique Albertini, Libération, 11/01/13 (en ligne : http://www.liberation.fr/economie/2013/01/11/ps-etouvriers-on-est-passe-de-l-abandon-au-mepris_873050, consulté le 15/08/14).

SALMON, C. (2007) : Storytelling, la machine à fabriquer des histoires et à formater les esprits, Paris, Éd. La Découverte.

WEINRICH, H. (2014 [1966]) : Linguistique du mensonge [Linguistik der Lüge], trad. de l'allemand, Limoges, Lambert-Lucas.

\section{NOTES}

1. En ligne : http://www.acrimed.org/article1688.html, consulté le 15/08/14.

2. En ligne: http://www.ipsos.fr/decrypter-societe/2014-01-21-nouvelles-fractures-francaisesresultats-et-analyse-l-enquete-ipsos-steria, consulté le 15/08/14.

3. L'École de Chicago est un groupe informel d'économistes libéraux généralement associés à Milton Friedman, à la théorie néoclassique des prix, au libre marché, au monétarisme, à une économie de l'offre opposée au fordisme et au keynésianisme (économie de la demande). Ce nom vient du département d'économie de l'université de Chicago dont la majorité des professeurs étaient membres de cette école. Elle rejoint l'École autrichienne de Friedrich Hayek pour qui la meilleure garantie de maintien d'une société civilisée réside dans un "ordre spontané» d'interaction qui seul permet « la mise en ordre de l'inconnu ». D'après Hayek, tenter d'imposer un ordre planifié par un petit nombre ne peut que détruire la responsabilité nécessaire à la régulation de l'ordre social. En d'autres termes, moins l'État intervient, mieux l'économie et la société se portent.

4. Toujours en 2011 , le revenu annuel moyen des $10 \%$ des déclarants les moins favorisés était de $8000 €$ ( $667 €$ par mois), tandis que celui des $10 \%$ les plus favorisés était de $58700 €$ - un rapport de 1 à 7,34 .

5. Je laisse de côté quelque $3 \%$ d'actifs agriculteurs à la composition de classe elle aussi très contrastée, avec les petits paysans d'un côté et les grands céréaliers de l'autre, en passant par les éleveurs, viticulteurs, horticulteurs, maraichers, producteurs de fruits et légumes, aux situations diverses selon l'étendue et la productivité des terres exploitées.

6. Le revenu plafond de la PPE est de $1462 €$ nets par mois et par personne, supérieur au salaire minimum interprofessionnel de croissance (Smic) qui est de $1129 €$ nets par mois (juillet 2014).

7. Selon Tite-Live, Histoire romaine, fable attribuée à Ésope racontée au peuple romain par Agrippa Menenius, en 494 avant J.-C., lors de troubles sociaux qui opposèrent plébéiens et patriciens : «Les membres du corps humain, voyant que l'estomac restait oisif, séparèrent leur cause de la sienne, et lui refusèrent leur office. Mais cette conspiration les fit bientôt tomber euxmêmes en langueur ; ils comprirent alors que l'estomac distribuait à chacun d'eux la nourriture qu'il avait reçue, et rentrèrent en grâce avec lui. Ainsi le sénat et le peuple, qui sont comme un seul corps, périssent par la désunion, et vivent pleins de force par la concorde». 
8. Je relève encore ceci dans Le Point, qui confirme la spécialisation du singulier dans le sens marxiste du terme : «Au Brésil, depuis dix ans, près de 40 millions de personnes ont rejoint la classe moyenne, qui représente désormais $52 \%$ de la population totale. Inexistante il y a vingt ans, la classe moyenne chinoise est aujourd'hui estimée à 157 millions » (Delhommais, 2013).

9. Selon L. Chauvel (2014) citant J. Damon (2013), les classes moyennes françaises sont ellesmêmes désormais «tirées vers le bas " par le déclassement scolaire, la précarité de l'emploi, la stagnation des salaires et la crise du logement. Selon les mêmes auteurs, la classe moyenne américaine " rétrécit » (on parle à son sujet de "shrinking middle class»), passant de $37 \%$ des ménages en 1974 à $30 \%$ en 2005, $28 \%$ en 2010 ; son revenu global est tombé de $60 \%$ à $45 \%$ du PIB entre 1970 et 2010 . Le renforcement des écarts de revenu et de bien-être entre les plus pauvres et les plus riches entraine une repolarisation (dualisation) de la société.

10. Mis en ligne en 2012 sur le site de Terra Nova. Voir aussi la réponse de B. Rothé (2013a, 2013b). B. Rothé note entre autres que le mot ouvrier ne compte que 15 occurrences dans les motions finales du dernier congrès du Parti socialiste.

11. Le Rapport Ipsos/Steria déjà cité dans mon introduction confirme cette situation: "Pour $68 \%$ (+7) [en janvier 2014 par rapport à janvier 1973] des cadres, la mondialisation est une opportunité alors qu'elle est perçue comme une menace par $74 \%(+4)$ des ouvriers. De même, pour près de trois cadres sur quatre $(72 \%,+16)$, la France doit s'ouvrir davantage au monde d'aujourd'hui alors que $75 \%(+13)$ des ouvriers pensent qu'elle doit s'en protéger. La coupure avec le politique est encore plus nette dans les classes populaires. $87 \%$ des ouvriers pensent que le système démocratique fonctionne mal et que leurs idées ne sont pas bien représentées $(+8)$, alors que cette idée est stable chez les cadres à $65 \%$ (+1). La demande d'autorité est beaucoup plus forte chez les ouvriers : $64 \%$ sont favorables au rétablissement de la peine de mort (contre $26 \%$ des cadres). À noter également que $74 \%$ des ouvriers estiment qu'on ne se sent plus chez soi comme avant $(+7$, contre $38 \%,-12$ chez les cadres) et que pour $39 \%$, les immigrés qui s'installent en France prennent le travail des Français (+ 7, contre $9 \%$ - 3 chez les cadres). »

12. Les professions intermédiaires représentent $23 \%$ de l'électorat total, contre $15 \%$ pour les classes moyennes supérieures.

\section{RÉSUMÉS}

Depuis les années 1980, l'École de Chicago inspire des politiques économiques qui ont discrédité la sociologie des classes sociales et des luttes de classes au profit de la collaboration entre catégories et groupes définis par le niveau de revenu. Le syntagme "classe ouvrière " a été remplacé par «classes moyennes » dans le discours des médias. Parallèlement, l'assimilation de la classe ouvrière à ce qui n'est pas «les classes moyennes blanches" ainsi que le rejet des immigrés et des enfants d'immigrés ont déplacé les luttes sociales vers les conflits ethniques (émeutes en banlieues, vocations djihadistes). Cette étude critique de lexicologie sociale montre comment les médias français, propriétés de grands groupes financiers, présentent une vision erronée des réalités sociales et contribuent à dépolitiser l'opinion publique sans égard pour l'éthique qui devrait fonder la vie démocratique du pays.

Since 1980, the Chicago school economics is inspiring policies that discredit the social classes and social struggles sociology for the benefit of collaboration between income-defined categories or groups. The words "working class" have been replaced by "middle classes" in massmedia 
discourse. At the same time, the assimilation of the working class with what is not White middle classes and the rejection of migrant workers and their sons moved social struggles into ethnic struggles (suburbs riots, djihadist vocations). This critical study in social lexicology shows how the French press, controlled by big bankers, is lying about social realities and contribute to depoliticize the country with no regards for ethics on which the democratic life should be based.

\section{INDEX}

Mots-clés : classe ouvrière, prolétariat, classe sociale, catégorie sociale, classes populaires, petite-bourgeoisie, bourgeoisie, travailleurs, travailleurs immigrés, clandestins sans-papiers, sociologie de Chicago, sociologie de presse, luttes sociales, luttes ethniques

Keywords : working class, proletariat, social class, social category, popular classes, middle-class, lower and upper middle class, bourgeoisie, workers, migrant workers, illegal workers, Chicago school sociology, journalistic sociology, social struggles, ethnic struggles

\section{AUTEUR}

\section{MARC ARABYAN}

CeReS (EA 3648), Université de Limoges 